\title{
Surface Damage Is Not Reduced With Highly Crosslinked Polyethylene Tibial Inserts at Short-term
}

\author{
Tong Liu MD, Christina Esposito PhD, Marcella Elpers BS, \\ Timothy Wright PhD
}

Published online: 18 June 2015

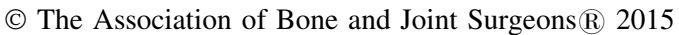

\begin{abstract}
Background Highly crosslinked ultrahigh-molecularweight polyethylene (XLPE) has been shown to reduce wear in hip arthroplasty, but the advantages over conventional polyethylene (PE) in total knee arthroplasty (TKA), if any, remain unclear.

Questions/purposes Do differences exist in (1) surface damage as measured by damage score and percent area affected; and (2) extent and location of dimensional changes between XLPE and conventional PE observed on retrieved TKA tibial inserts?
\end{abstract}

\footnotetext{
One of the authors (TL) received funding from the China Scholarship Council (Award Number 201306170105). One of the authors (CE) is partially supported by a Ruth L. Kirschstein NRSA from the National Institute of Arthritis and Musculoskeletal and Skin Diseases of the National Institutes of Health under Award Number AR007281. The content is solely the responsibility of the authors and does not necessarily represent the official views of the National Institutes of Health. The present research was financially supported by the Mary and Fred Trump Institute for Implant Analysis (TW, DP).

All ICMJE Conflict of Interest Forms for authors and Clinical Orthopaedics and Related Research ${ }^{\circledR}$ editors and board members are on file with the publication and can be viewed on request.

Clinical Orthopaedics and Related Research ${ }^{\mathbb{B}}$ neither advocates nor endorses the use of any treatment, drug, or device. Readers are encouraged to always seek additional information, including FDAapproval status, of any drug or device prior to clinical use.

Each author certifies that his or her institution approved the human protocol for this investigation, that all investigations were conducted in conformity with ethical principles of research, and that informed consent for participation in the study was obtained.

This work was performed at the Hospital for Special Surgery, New York, NY, USA.
}

T. Liu ( $₫)$, C. Esposito, M. Elpers, T. Wright

Department of Biomechanics, Hospital for Special Surgery,

535 East 70th Street, New York, NY 10021, USA

e-mail: liut@hss.edu; liutong1986@gmail.com
Methods In this study of components retrieved at the time of revision surgery, we matched 44 XLPE to 44 conventional PE inserts from four manufacturers; the matching approach considered implant design (exact match), insert size (exact match), and length of implantation (matched \pm 6 months). Surface damage on the articular surfaces was subjectively graded and digitally mapped to determine the percent damaged area of each damage mode. Three-dimensional changes that had occurred as a result of implantation were determined by comparing laser scans of the retrieved inserts with size-matched pristine inserts.

Results The differences of damage scores and percent damaged areas between the matched XLPE and conventional PE inserts were not large enough to be clinically significant with low corresponding levels of statistical significance (scores: $42 \pm 13 ; 95 \%$ confidence interval $[\mathrm{CI}], 38-46$ versus $45 \pm 13 ; 95 \% \mathrm{CI}, 41-49 ; \mathrm{p}=0.4$; percent areas: $54 \% \pm 38 \%$; 95\% CI, $44 \%-64 \%$ versus $54 \% \pm 32 \% ; 95 \% \mathrm{CI}, 42 \%-65 \% ; \mathrm{p}=0.9)$. However, XLPE inserts showed greater articular surface dimensional changes with high significance (root mean square of the distance: $0.16 \pm 0.06 \mathrm{~mm}$; 95\% CI, 0.13-0.18 mm versus $0.14 \pm 0.05 \mathrm{~mm} ; 95 \%$ CI, $0.11-0.16 \mathrm{~mm} ; \mathrm{p}=0.03$ ). Within the same design, deviation patterns were consistent between the two materials; however, as expected, the location of the dimensional changes differed among designs: the negative deviations on the plateaus were centrally located in Zimmer PS inserts, were located on the perimeter in Smith \& Nephew PS inserts, and were across the entire surface in DePuy PS inserts.

T. Liu

Department of Orthopaedics, China-Japan Union Hospital of Jilin University, Changchun, Jilin, China 
Conclusions We found no difference in surface damage between matched XLPE and conventional PE inserts of the same designs. However, increased dimensional changes in TKAs with XLPE may reflect larger contact areas and potentially explain improved performance of XLPE in published simulator studies.

Clinical Relevance The lack of meaningful differences between the two polyethylene materials suggests caution in adopting a new, more expensive bearing material over another material that has a long track record of excellent behavior. A possible advantage is the greater dimensional changes, which could be the result of the lower creep resistance of XLPE, but this advantage awaits long-term results.

\section{Introduction}

Highly crosslinked ultrahigh-molecular-weight polyethylene (XLPE) has demonstrated superior wear performance compared with conventional polyethylene (PE) in THA with followup studies beyond 10 years showing few or no patients with osteolysis [5, 6, 10, 21, 24, $26,32]$. XLPE was more recently introduced into TKA after in vitro knee simulator findings of lower wear rates compared with those found with conventional PE [12, 22, 33, 34]. Thus far, however, the clinical benefit of XLPE remains unclear. For example, the Kaiser Implant Registry [16] with 62,177 primary TKAs at followups of 1 to 5 years showed no difference in revision surgery between XLPE and conventional PE bearing surfaces. In contrast, the 2014 Annual Report of the Australian Orthopaedic Association National Joint Replacement Registry [1] found primary TKAs with XLPE had a lower rate of revision than those with conventional $\mathrm{PE}$, although this result was confounded by the much smaller amount of XLPE in use and variations in implant design.

Additional concerns exist for the use of XLPE in TKA. The larger stresses and more complex stress distributions in tibial TKA PE components compared with PE acetabular components in THA [3, 4] must be considered in light of the alterations in material properties caused by the crosslinking process [2, 29]. For example, the reduced fracture toughness in XLPE is a cause for concern in tibial post fractures in posterior-stabilized TKAs [30]. Evaluation of surface damage and oxidative properties of retrieved XLPE TKA tibial components from revision surgeries showed little improvement with the use of XLPE [20, 27]. The first retrieval analysis of XLPE tibial inserts used an optical grading method to analyze surface damage and found no differences between eight XLPE tibial inserts and 71 conventional PE inserts [23]. However, a major limitation of these retrieval studies is the lack of controlled conditions across study groups. Implant design and length of implantation are important variables that may bias a comparison of the benefit of XLPE and conventional PE tibial components based on materials alone. Also, none of the investigations on XLPE damage were done in three dimensions, and differences in damage patterns between materials were not examined.

Therefore, we designed a matched-pair analysis of components retrieved at revision surgery and used the techniques of damage scoring, damage mapping, and threedimensional (3-D) laser scanning to ask the following questions: (1) is XLPE more damage-resistant than conventional PE inserts in TKA based on damage score and percent area affected; and (2) does XLPE differ in extent and location of dimensional changes as measured with 3-D laser scanning compared with conventional PE?

\section{Patients and Methods}

In this study of components retrieved at the time of revision surgery, we matched 44 knees with XLPE tibial inserts to 44 knees with conventional PE inserts using the following matching conditions: implant design (exact match of 39 posterior-stabilized, three cruciate-retaining, and two constrained condylar), insert size (exact match), and length of implantation (LOI, matched \pm 6 months). There were four pairs of DePuy (DePuy Inc, Warsaw, IN, USA), 13 pairs of Smith \& Nephew (Smith \& Nephew Plc, London, UK), four pairs of Stryker (Stryker Corp, Kalamazoo, MI, USA), and 23 pairs of Zimmer (Zimmer Inc, Warsaw, IN, USA) inserts. The resins, sterilization modalities, crosslinking modalities, and postirradiation processes for the XLPE and conventional PE inserts differed among the implants (Tables 1, 2). Demographic and clinical data were collected from medical records. No differences were found in the reasons for revision between the two polyethylene materials (Table 3). No differences existed in body mass index (BMI), age, or sex between the patients whose components were paired (Table 4). Implants were matched on insert size, but not insert thickness; nonetheless, no difference was found between the thicknesses of the XLPE (12 $\pm 4 \mathrm{~mm}$; range, $8-25 \mathrm{~mm}$ ) and conventional $\mathrm{PE}$ (11 $\pm 2 \mathrm{~mm}$; range, 9-20 mm) inserts $(\mathrm{p}=0.19)$.

For 29 pairs (four pairs of DePuy, 10 pairs of Smith \& Nephew, and 15 pairs of Zimmer TKAs), pristine, neverimplanted TKA conventional PE tibial inserts were available in our laboratory to match the designs and sizes of the retrievals. Funding for the study did not permit the purchase of matching pristine inserts for the additional 15 pairs. 
Table 1. The implant properties of the XLPE inserts

\begin{tabular}{|c|c|c|c|c|c|}
\hline Insert design (XLPE name) & Resin & $\begin{array}{l}\text { Sterilization } \\
\text { modality }\end{array}$ & Crosslinking modality & $\begin{array}{l}\text { Postirradiation } \\
\text { process }\end{array}$ & $\begin{array}{l}\text { Number } \\
(\%)\end{array}$ \\
\hline DePuy Sigma PS (XLK) & $\begin{array}{l}\text { GUR } \\
\quad 1020\end{array}$ & Gas plasma & Gamma irradiation $50 \mathrm{kGy}$ & $\begin{array}{l}\text { Remelted and } \\
\text { annealed }\end{array}$ & $4(9.1)$ \\
\hline $\begin{array}{l}\text { Smith \& Nephew Gen II/Legion PS High } \\
\text { Flex (XLPE) }\end{array}$ & $\begin{array}{l}\text { GUR } \\
1020\end{array}$ & Ethylene oxide & Gamma irradiation $75 \mathrm{kGy}$ & Remelted & $13(29.5)$ \\
\hline Stryker Triathlon CR (X3) & $\begin{array}{l}\text { GUR } \\
\quad 1020\end{array}$ & Gas plasma & $\begin{array}{l}\text { Gamma irradiation } 30 \mathrm{kGy} \text { in } 3 \text { steps } \\
\text { (90 kGy total) }\end{array}$ & $\begin{array}{l}\text { Sequential } \\
\text { annealed }\end{array}$ & $2(4.5)$ \\
\hline Stryker Triathlon PS (X3) & $\begin{array}{l}\text { GUR } \\
1020\end{array}$ & Gas plasma & $\begin{array}{l}\text { Gamma irradiation } 30 \mathrm{kGy} \text { in } 3 \text { steps } \\
\text { (90 kGy total) }\end{array}$ & $\begin{array}{l}\text { Sequential } \\
\text { annealed }\end{array}$ & $2(4.5)$ \\
\hline Zimmer Legacy PS High Flex (Prolong) & $\begin{array}{l}\text { GUR } \\
1050\end{array}$ & Gas plasma & Electron beam irradiation $65 \mathrm{kGy}$ & Remelted & $20(45.4)$ \\
\hline Zimmer NexGen CR (Prolong) & $\begin{array}{l}\text { GUR } \\
\quad 1050\end{array}$ & Gas plasma & Electron beam irradiation $65 \mathrm{kGy}$ & Remelted & $1(2.5)$ \\
\hline Zimmer Legacy CCK (Prolong) & $\begin{array}{l}\text { GUR } \\
1050\end{array}$ & Gas plasma & Electron beam irradiation $65 \mathrm{kGy}$ & Remelted & $2(4.5)$ \\
\hline
\end{tabular}

$\overline{\mathrm{XLPE}}=$ highly crosslinked ultrahigh-molecular-weight polyethylene; $\mathrm{PS}=$ posterior-stabilized; $\mathrm{CR}=$ cruciate-retaining; $\mathrm{CCK}=$ constrained condylar.

Table 2. The implant properties of the conventional PE inserts

\begin{tabular}{lllc}
\hline Insert design & Resin & Sterilization modality & Number (\%) \\
\hline DePuy Sigma PS & GUR 1020 & Gamma vacuum foil sterilization $(\mathrm{GVF})$ & $4(9.1)$ \\
Smith \& Nephew Gen II/Legion PS High Flex & GUR 1020 & Ethylene oxide & 13 (29.5) \\
Stryker Triathlon CR & GUR 1020 & Gamma sterilization in nitrogen & $2(4.5)$ \\
Stryker Triathlon PS & GUR 1020 & Gamma sterilization in nitrogen & $2(4.5)$ \\
Zimmer Legacy PS High Flex & GUR 1050 & Gamma sterilization in nitrogen & 20 (45.4) \\
Zimmer NexGen CR & GUR 1050 & Gamma sterilization in nitrogen & $1(2.5)$ \\
Zimmer Legacy CCK & GUR 1050 & Gamma sterilization in nitrogen & $2(4.5)$ \\
\hline
\end{tabular}

$\mathrm{PE}=$ polyethylene; $\mathrm{PS}=$ posterior-stabilized; $\mathrm{CR}=$ cruciate-retaining; $\mathrm{CCK}=$ constrained condylar.

Table 3. Reasons for revision for conventional polyethylene and highly crosslinked ultrahigh-molecular-weight polyethylene retrieved inserts

\begin{tabular}{lccc}
\hline Indication for revision & Conventional insert & Crosslinked insert & p value \\
\hline Stiffness & $7(16 \%)$ & $14(32 \%)$ & 0.3 \\
Instability & $11(25 \%)$ & $12(27 \%)$ & 0.3 \\
Loosening & $7(16 \%)$ & $8(18 \%)$ & 0.3 \\
Infection & $12(27 \%)$ & $7(16 \%)$ & 0.3 \\
Other & $7(16 \%)$ & $3(7 \%)$ & 0.3 \\
\hline
\end{tabular}

Comparison was made using Pearson's chi-square test.

\section{Visual Assessment of Damage}

The articular surfaces of the inserts were divided into 15 regions for a posterior-stabilized or constrained condylar design and into 10 regions for a cruciate-retaining design (Fig. 1). Burnishing, pitting, scratching, delamination, surface deformation, abrasion, and third-body debris were subjectively graded on a scale of 0 to 3 to reflect the extent and severity of each damage mode [15]. The maximum total damage score was 315 for a posterior-stabilized insert surface and 210 for a cruciate-retaining insert surface. Two independent observers (TL, CE) visually assessed damage on the articular surfaces in each region using a light stereomicroscope $(\times 10-\times 32$ magnification). If the difference in total damage scores summed across all regions was $>10$ points between the two graders, then a third observer (ME) scored the insert as well (this occurred in 10 [11\%] of the 88 inserts). 
Table 4. Demographic data for the patients from whom the conventional PE and XLPE inserts were retrieved

\begin{tabular}{llll}
\hline Variable & Conventional insert & Crosslinked insert & p value \\
\hline Number & 44 & 44 & N/A \\
Number of females $(\%)$ & $29(66 \%)$ & $25(57 \%)$ & 0.4 \\
Length of implantation (months) & $15 \pm 9$ (range, 1-44) & $15 \pm 8$ (range, 4-44) \\
Body mass index $\left(\mathrm{kg} / \mathrm{m}^{2}\right)$ & $30 \pm 5$ (range, 18-43) & $31 \pm 6$ (range, 19-45) & 0.9 \\
Age at revision (years) & $66 \pm 10$ (range, 48-89) & $63 \pm 9$ (range, 44-81) & 0.7 \\
\hline
\end{tabular}

Data are presented as mean $\pm \mathrm{SD}$; comparisons were made using paired Student's t-tests for continuous variables and chi square tests for categorical variables; $\mathrm{PE}=$ polyethylene; XLPE = highly crosslinked ultrahigh-molecular-weight polyethylene; N/A $=$ not applicable .

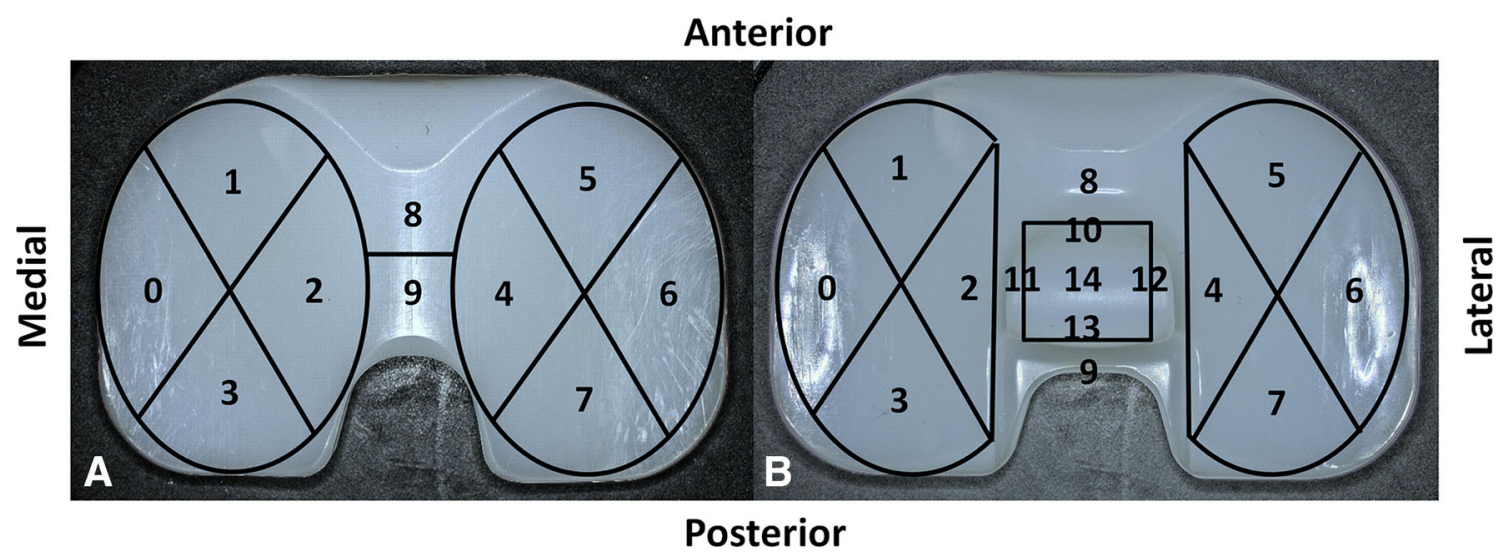

Fig. 1A-B The tibiofemoral articular surface was divided into 10 sections for the cruciate-retaining designs (A) and into 15 sections for the posterior-stabilized design (B).

Articular surface damage maps of the medial and lateral plateaus were created to calculate the percent area affected by each damage mode. The superior surface of each insert was digitally photographed using a Keyence microscope (Keyence Corp, Osaka, Japan) using the high-dynamic range mode. The damage modes that had been identified using the light stereomicroscope were then manually replicated onto the digital photos using Photoshop CS2 software (Adobe Systems, San Jose, CA, USA). Each damage mode was drawn as an individual layer, and the coverage area of each layer was subsequently analyzed using ImageJ (NIH, Bethesda, MD, USA).

\section{Measuring Surface Dimensional Change}

Changes to the dimensions of the articular surfaces of the retrieved implants were estimated by direct comparison to the corresponding pristine (never implanted) inserts. The 29 pairs of retrieved inserts with conventional PE pristine implants available were all scanned using a desktop 3-D laser scanner (Range 7; Konica Minolta, Inc, Tokyo, Japan; accuracy $=40 \mu \mathrm{m}$ ). Aerosol talc was used to coat the inserts. Sixteen scans of different views of each insert were taken to obtain the entire surface. Eight of the scans were taken with the surface of insert at an inclination of $45^{\circ}$ from the scanner beam (Fig. 2A), and another eight were taken at $135^{\circ}$ from the beam (Fig. 2B). All scans were taken at every $45^{\circ}$ of revolution.

The resulting point clouds from the scans of each insert were aligned and combined using Geomagic Studio software (Version 12; Morrisville, NC, USA) to convert the scans into a single 3-D polygon model. The 3-D models of the size-matched pristine inserts were obtained using the same procedure. The 3-D reconstruction of a retrieved insert was aligned in Geomagic to the size-matched 3-D reconstruction of the corresponding pristine insert. The alignments were according to the backside surfaces of both inserts and the surrounding noncontact surfaces around the post for posterior-stabilized designs. For cruciate-retaining designs, we used the backside and noncontact articular surfaces. These noncontact surfaces were used for alignment to reduce error from backside wear in the retrieved tibial inserts and from manufacturing tolerances in insert thicknesses. Once the reconstructions were aligned, the average distance, or "deviation," between the surfaces of the retrieved and pristine inserts was measured between closest point pairs in the two point clouds. This approach 


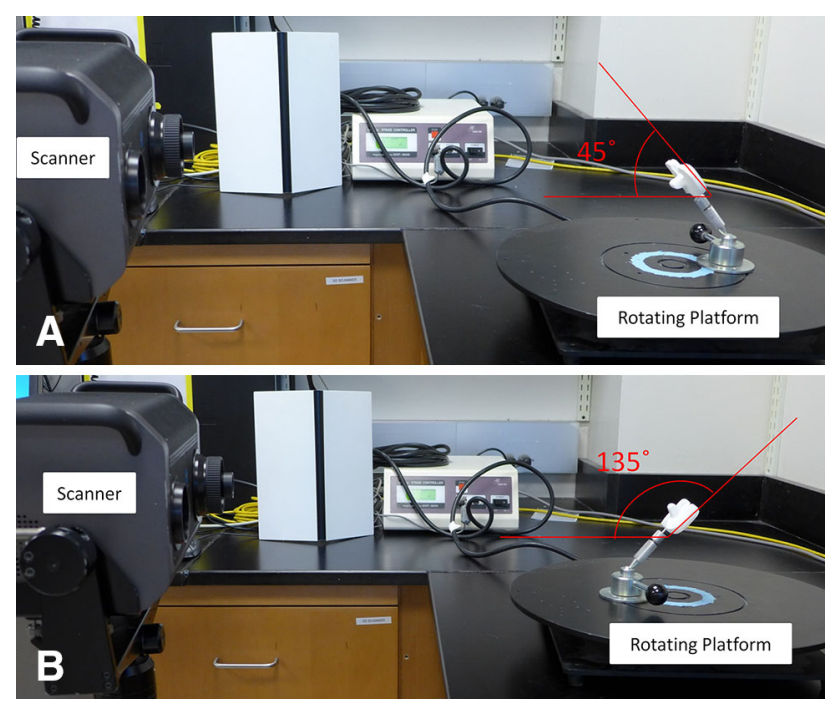

Fig. 2A-B Each insert was scanned 16 times to obtain the entire surface. Eight scans were taken with the surface of the insert inclined $45^{\circ}$ to the scanner beam (A), and another eight scans were taken at $135^{\circ}$ from the beam (B). The rotating platform turned $45^{\circ}$ clockwise after each scan to achieve the total geometry of each insert.

was reported previously to demonstrate the extent of dimensional change in tibial insert surfaces [31].

The word "deviation" was used because we do not know if the differences were the result of removal of the material, deformation, or a combination of the two. However, in the deviation calculation, positive and negative values can be neutralized. Therefore, we also applied the root mean square of the distance (RMSd) between retrieval and pristine point clouds as a reliable indicator of the variability when applied on a surface with obvious dimensional changes:

$R M S d=\sqrt{\frac{\sum_{i} d_{i}^{2}}{n}}$ where $d$ is the distance between each of the $n$ pairs of closest points between the two articular surfaces. The volume difference between the articular surfaces of the plateaus of the retrieved and pristine inserts was also calculated based on a fixed reference plane (the backside plane of the pristine insert). If the articular surface of the retrieved component was above the pristine insert surface (which may be found if the PE were to swell or to be pushed up at the edges of the contact area), the volume value was positive, and if it was below, the volume value was negative (as a result of wear and/or deformation).

\section{Statistical Analysis}

For matching XLPE and conventional PE retrievals, patient BMI and age at revision were compared using a paired Student's t-test, and the sex of the patients was compared using a chi-square test. A Wilcoxon signed-rank test was used to compare the differences in the thicknesses between the XLPE and conventional PE inserts. Polyethylene damage scores and the percent damaged areas of XLPE and conventional PE inserts were compared using a paired t-test for parametric data (the burnishing, scratching, pitting, and total damage scores; the percent area of burnishing in the damage maps) and a Wilcoxon signed-rank test for nonparametric data (the deformation, third-body debris, and abrasion damage scores as well as the scores for each damage mode in the post area; the percent area of pitting, scratching, deformation, embedded third-body debris, and abrasion in the damage maps). Differences in the average distance and RMSd between the two materials were also calculated with a Wilcoxon signed-rank test because the data were nonparametric. The level of statistical significance corresponding to the observed differences in volume change of the bearing surfaces was calculated with a paired t-test. Analyses were done to determine the strength of the correlations between demographic data (age, BMI, LOI) and the damage scores, percent damaged areas, and the volume changes of the bearing surfaces. Pearson's coefficient and Spearman's rank order coefficients were applied depending on the distribution of the data.

\section{Results}

The differences between the damage scores for the matched XLPE and conventional PE inserts were not large enough to be clinically significant with a low corresponding level of statistical significance for all of the total combined score for all the damage modes (42 $\pm 13 ; 95 \%$ CI, 38-46 for XLPE versus $45 \pm 13$; 95\% CI, 41-49 for conventional $\mathrm{PE} ; \mathrm{p}=0.4$ ) except for third-body debris, which was more prevalent in conventional $\mathrm{PE}$ inserts $(0.1 \pm 0.4 ; 95 \%$ CI, $0-0.3$ versus $0.4 \pm 0.7$; $95 \%$ CI, $0.2-$ $0.6 ; \mathrm{p}=0.04$; Table 5). The average total damage scores for XLPE and conventional PE inserts within each manufacturer were $34 \pm 25$ (range, 10-69) and $51 \pm 19$ (range, 28-78; $\mathrm{p}=0.2)$ for DePuy, $48 \pm 11$ (95\% CI, 26-66) and $44 \pm 10(95 \%$ CI, 17-58; $\mathrm{p}=0.5)$ for Smith \& Nephew, $40 \pm 9$ (range, 33-53) and $45 \pm 22$ (range, 28-78; $\mathrm{p}=0.7)$ for Stryker, and $41 \pm 12$ (95\% CI, 31-60) and $44 \pm 12$ (95\% CI, 28-71; p = 0.3) for Zimmer, respectively. Delamination was not observed in any of the inserts. No cracks were visible on any of the posts in the posteriorstabilized knees. No relationship was found between damage scores and LOI for inserts made of either type of PE. The differences in the percent of the area of the articular surfaces that was damaged were not large enough to be clinically significant between the XLPE and conventional PE insert pairs for any of the damage modes with a low corresponding level of statistical significance 
Table 5. Average damage scores for each of the seven damage modes on the articular surfaces of the XLPE and conventional PE (Conv-PE) inserts

\begin{tabular}{|c|c|c|c|c|c|c|c|c|c|}
\hline \multirow[t]{2}{*}{ Damage mode score } & \multicolumn{3}{|l|}{ Plateaus } & \multicolumn{3}{|l|}{ Post } & \multicolumn{3}{|l|}{ Total } \\
\hline & XLPE & Conv-PE & $\mathrm{p}$ value & XLPE & Conv-PE & $\mathrm{p}$ value & XLPE & Conv-PE & $\mathrm{p}$ value \\
\hline Burnishing & $14 \pm 8$ & $14 \pm 8$ & 0.6 & $1 \pm 2$ & $2 \pm 2$ & 0.2 & $15 \pm 8$ & $15 \pm 9$ & 0.9 \\
\hline Pitting & $11 \pm 5$ & $11 \pm 6$ & 0.7 & $0.2 \pm 0.6$ & $0.5 \pm 1.1$ & 0.2 & $11 \pm 5$ & $12 \pm 6$ & 0.6 \\
\hline Scratching & $14 \pm 6$ & $14 \pm 5$ & 0.4 & $0.6 \pm 0.7$ & $0.8 \pm 1.5$ & 0.6 & $14 \pm 6$ & $15 \pm 6$ & 0.3 \\
\hline Third-body debris & $0.1 \pm 0.4$ & $0.3 \pm 0.6$ & 0.09 & $0.0 \pm 0.2$ & $0.1 \pm 0.3$ & 0.2 & $0.1 \pm 0.4$ & $0.4 \pm 0.7$ & 0.04 \\
\hline Abrasion & $0.3 \pm 0.5$ & $0.4 \pm 1.1$ & 0.5 & $0.0 \pm 0.3$ & $0.3 \pm 0.8$ & 0.09 & $0.3 \pm 0.6$ & $0.7 \pm 1.2$ & 0.07 \\
\hline Deformation & $0.4 \pm 1.0$ & $0.5 \pm 0.9$ & 0.4 & $1.0 \pm 1.6$ & $1.1 \pm 1.5$ & 0.6 & $1.4 \pm 1.8$ & $1.6 \pm 1.8$ & 0.4 \\
\hline Delamination & $0 \pm 0$ & $0 \pm 0$ & 1.0 & $0 \pm 0$ & $0 \pm 0$ & 1.0 & $0 \pm 0$ & $0 \pm 0$ & 1.0 \\
\hline Total & $39 \pm 12$ & $40 \pm 10$ & 0.7 & $3 \pm 3$ & $4 \pm 4$ & 0.08 & $42 \pm 13$ & $45 \pm 13$ & 0.4 \\
\hline
\end{tabular}

Data are presented as mean $\pm \mathrm{SD}$; comparisons were made using paired Student's t-tests or Wilcoxon signed-rank tests; XLPE $=$ highly crosslinked ultrahigh-molecular-weight polyethylene; $\mathrm{PE}=$ polyethylene.

Table 6. The percentages of the articular surface area covered by the damage modes are presented for XLPE and conventional PE inserts

\begin{tabular}{lccl}
\hline Percent damaged areas & \multicolumn{1}{c}{ XLPE } & Conventional PE & $\mathrm{p}$ value \\
\hline Burnishing & $41 \pm 23$ & $39 \pm 30$ & 0.8 \\
Pitting & $5 \pm 12$ & $6 \pm 7$ & 0.2 \\
Scratching & $7 \pm 13$ & $7 \pm 9$ & 0.1 \\
Abrasion & $0.1 \pm 0.5$ & $0.3 \pm 1.2$ & 0.3 \\
Third-body debris & $0.3 \pm 2.1$ & $0.1 \pm 0.2$ & 0.1 \\
Deformation & $0.5 \pm 1.1$ & $1.7 \pm 4.8$ & 0.1 \\
Delamination & $0 \pm 0$ & $0 \pm 0$ & 1.0 \\
\hline
\end{tabular}

Data are presented as mean $\pm \mathrm{SD}$; comparisons were made using paired Student's t-tests or Wilcoxon signed-rank tests; XLPE = highly crosslinked ultrahigh-molecular-weight polyethylene; $\mathrm{PE}=$ polyethylene.

$(54 \% \pm 38 \% ; 95 \% \mathrm{CI}, 44 \%-64 \%$ versus $54 \% \pm 32 \%$; $95 \%$ CI, $42 \%-65 \% ; \mathrm{p}=0.9$; Table 6$)$. In more than half of the inserts, burnishing was visible in the same areas where negative volume changes occurred in the corresponding 3-D deviation maps (Fig. 3).

The differences in mean distances and volume changes between XLPE and conventional PE inserts were not large enough to be clinically significant with low corresponding levels of statistical significance (mean distance: $0.13 \pm$ $0.05 \mathrm{~mm}$; 95\% CI, 0.11-0.15 mm versus $0.11 \pm 0.04 \mathrm{~mm}$; $95 \%$ CI, $0.10-0.13 \mathrm{~mm} ; \quad \mathrm{p}=0.15$; volume change: $-207 \pm 84 \mathrm{~mm}^{3} ; 95 \% \mathrm{CI},-175$ to $239 \mathrm{~mm}^{3}$ versus $-187 \pm 91 \mathrm{~mm}^{3} ; 95 \% \mathrm{CI},-152$ to $\left.221 \mathrm{~mm}^{3} ; \mathrm{p}=0.18\right)$. The RMSd in the XLPE insert group was greater than in the conventional PE group $(0.16 \pm 0.06 \mathrm{~mm} ; 95 \% \mathrm{CI}$, $0.13-0.18 \mathrm{~mm}$ versus $0.14 \pm 0.05 \mathrm{~mm}$; $95 \% \mathrm{CI}, 0.11-$ $0.16 \mathrm{~mm} ; \mathrm{p}=0.03$; Fig. 4). Linear correlations were demonstrated between LOI and the volume differences in both XLPE and conventional PE inserts (Fig. 5).
The locations of the dimensional changes on the articular surfaces were consistent within designs; however, the location of dimensional changes varied among designs: retrieved Zimmer posterior-stabilized inserts had negative deviations centrally located on the plateaus, Smith \& Nephew posterior-stabilized inserts had negative deviations located on the perimeter of the plateaus, and DePuy posterior-stabilized inserts had negative deviations on the entire surface of the plateaus (Fig. 6).

\section{Discussion}

The wear resistance of ultrahigh-molecular-weight PE can be increased by introducing crosslinking into the molecular architecture [28]. The improvement in wear performance in XLPE tibial inserts is supported by wear simulator experiments [12, 22, 33, 34], but evidence of in vivo success is required for a comprehensive assessment. In our study, retrieval analysis was used to compare the accumulation of damage in vivo of conventional and highly crosslinked PE tibial plateaus. We combined damage scoring, a wellestablished method for comparing the amount of accumulated damage in retrieved PE implants [15], with damage mapping [8] and 3-D laser scanning [24] to evaluate surface damage and dimensional change of retrieved tibial inserts. Damage mapping allowed us to measure the area percent and evaluate the distribution of each damage mode on the articular surface of the inserts, whereas 3-D laser scanning measured the depth of penetration (deformation plus wear) into the articular surface. Together these modalities allowed us to address our research questions of damage resistance and extent and location of dimensional changes in XLPE inserts versus conventional PE inserts after relatively short in vivo periods. 


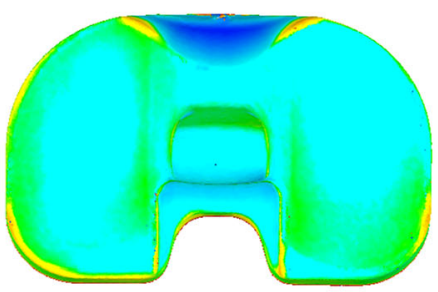

A

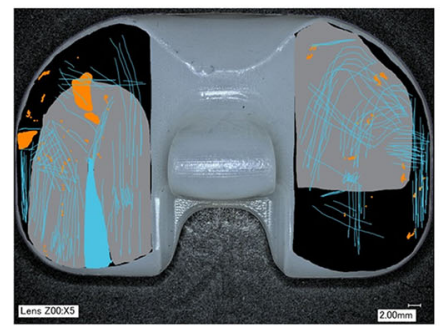

D
Fig. 3A-F Color maps of the 3-D deformations (A-C) and the corresponding damage maps (D-F) are shown for the articular surfaces of the same three inserts. Negative dimensional changes (the light blue area) of the plateaus on the 3-D maps coincide with the area

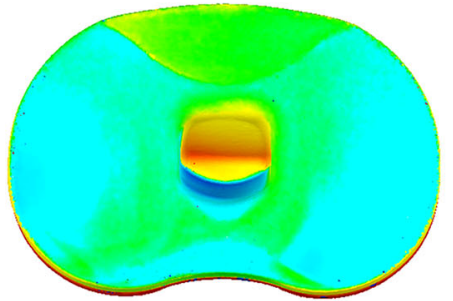

B

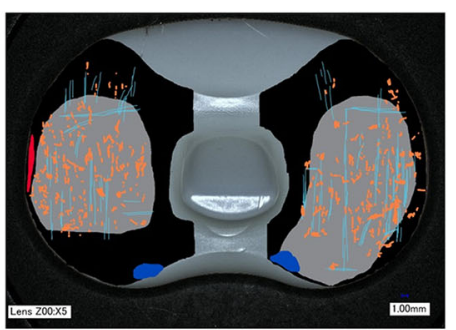

E

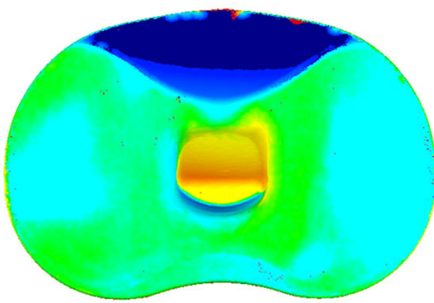

C

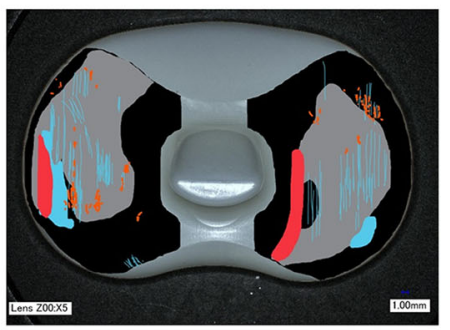

$\mathbf{F}$
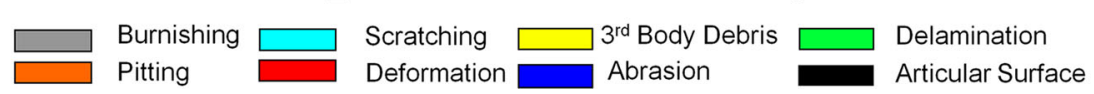

that was covered by burnishing (the gray area) on the damage maps. The scale bar on the right applies to $\mathbf{A}-\mathbf{C}$ with units in millimeters; the color codes in the damage maps indicate the seven damage modes (D-F).
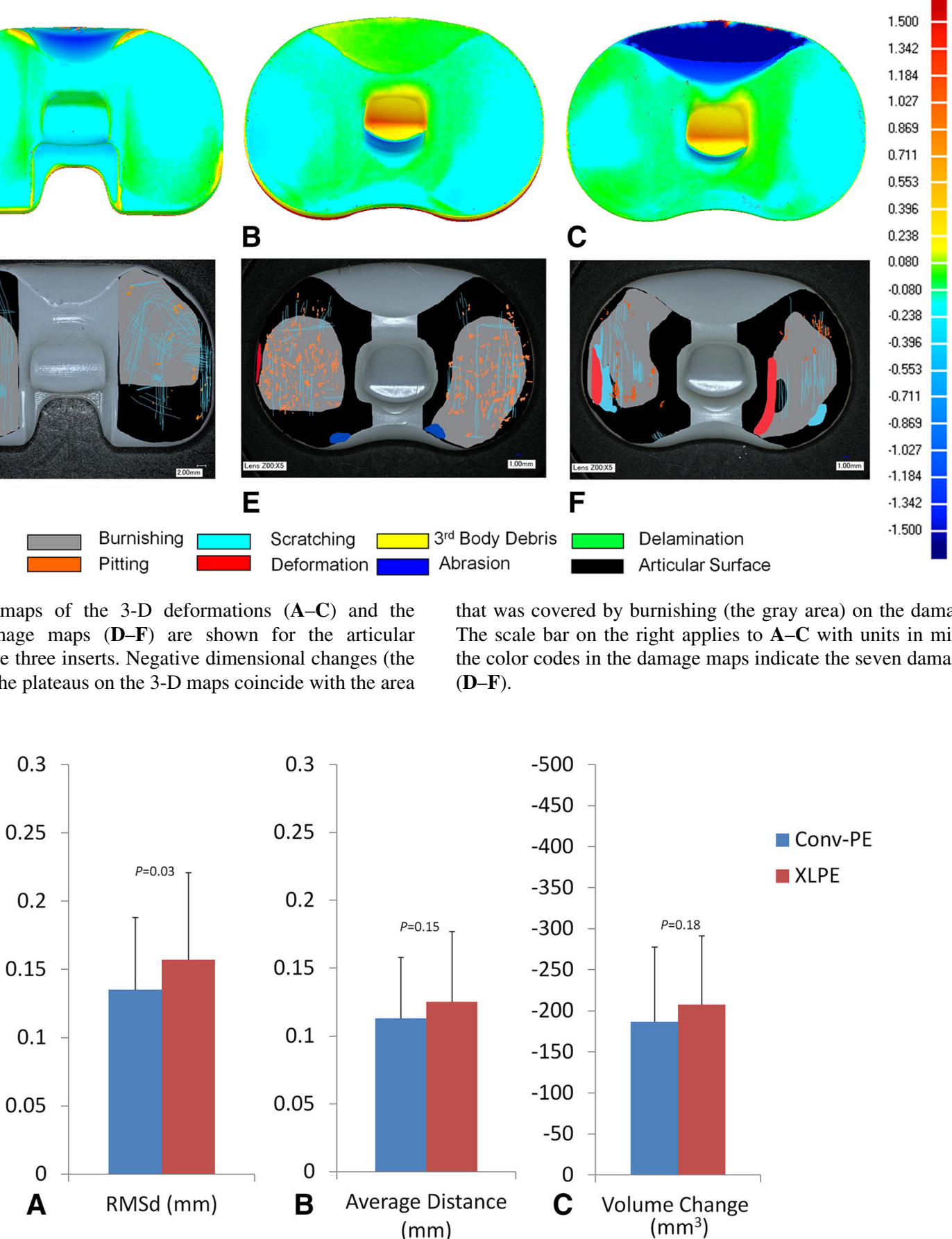

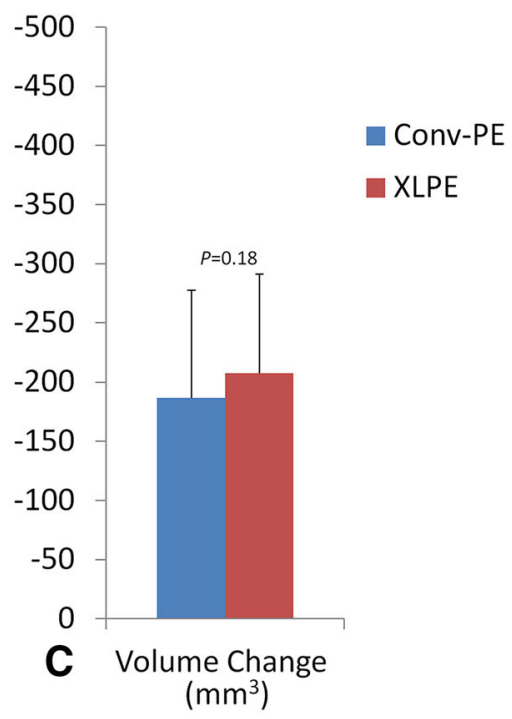

Fig. 4A-C (A) RMSd, (B) average distance, and (C) volume change bar charts are shown for the dimensional changes of the XLPE and conventional PE (Conv-PE) insert groups. Wilcoxon signed-rank test and paired Student's t-tests were used for the comparisons.

Limitations in our study must be considered. Inherent limitations of any retrieval study are that such studies are retrospective for which certain variables such as reason for revision cannot be controlled and that the cohort includes implants that by definition have failed and thus may not reflect well-functioning devices. However, we were able to use a large series of retrievals to match the manufacturer, implant design, and LOI, and in the current study, the differences between materials in revision indications did not reach statistical significance (Table 3). Another limitation is the short LOI (an average of 15 months) for the studied inserts. Future retrieval studies will need to see whether the no-difference findings in our report remain as such or whether indeed differences in surface damage 


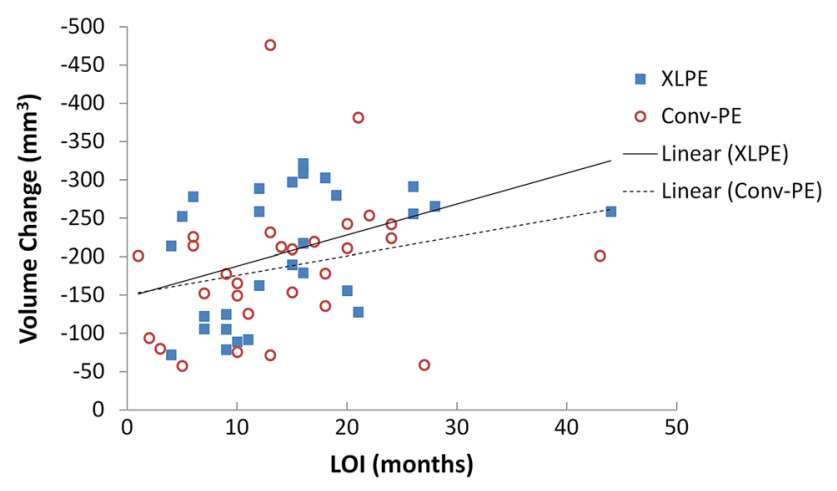

Fig. 5 Linear correlations were demonstrated between LOI and the volume changes in both the XLPE group $\left(R^{2}=0.17\right)$ and the conventional PE (Conv-PE) group $\left(\mathrm{R}^{2}=0.06\right)$.

between XLPE and conventional PE develop over time. A third limitation was the scanning of pristine inserts to determine the deviations in the retrieved inserts. We assumed the pristine conventional PE inserts had the same original surface geometries that our retrieved XLPE and conventional PE implants had before implantation. However, the manufacturing tolerances of the pristine and retrieved implants could have been large enough to influence our results. However, this limitation was unavoidable, because the original dimensions of the retrieved implants were unavailable. As a final limitation, the size-matched pristine implants in our study were conventional $\mathrm{PE}$, and we only had 29 of 44 pairs available for analysis. However, based on our 3-D scanning data, similar colorimetric maps were seen on the articular surfaces in both XLPE and conventional PE inserts. Therefore, we believe it acceptable to use conventional PE inserts in investigating the dimensional changes of the same design XLPE implants.

We found no short-term improvement in surface damage resistance with the use of XLPE in TKA tibial inserts. Burnishing, pitting, and scratching were the primary damage modes in both groups. Irradiation-induced crosslinking and postirradiation thermal treatments can lead to the reduction of fatigue resistance in XLPE [2, 14, 25, 29]. Therefore, we expected higher scores for fatigue-related damage modes (pitting, abrasion, and delamination) with XLPE inserts. However, the severity and extent of the pitting and abrasion were not different between the materials, and no delamination was found in any of the inserts. Although we found an increase in third-body debris for conventional PE inserts, this finding is likely to be clinically unimportant because the scores for both XLPE and conventional PE were so small (0.1 versus 0.4 , respectively).

We found greater dimensional change in XLPE inserts when considering the average depth distance (RMSd) between retrieved and pristine point clouds of the articular surfaces ( $p=0.03$; Fig. 4). A similar trend was found with larger burnished areas on the XLPE surfaces, but the differences were small ( $p=0.8$; Table 6$)$. This might be the result of the differences between damage mapping (a twodimensional measurement) and laser scanning (a 3-D measurement). Interestingly, the negative 3-D dimensional changes correlated with the burnished areas in damage mapping for more than half of our subjects (Fig. 3). The burnished contact surface is dependent on a patient's unique kinematics, the design characteristics of the TKA (for example, the conformity of the articular surface), and the PE properties. So, if two implants of the same size and design (one with XLPE and one with conventional PE) were in patients with similar kinematics, we could compare the resulting deformation of the PE materials alone. In the current study, we matched in size and design conventional $\mathrm{PE}$ and XLPE retrievals and found greater deformation on the XLPE surfaces.

XLPE is less creep-resistant than conventional PE [19], and knee simulator tests found XLPE inserts deformed more than conventional PE inserts [22]. The lower crystallinity and hence lower creep resistance results from crosslinking and subsequent remelting [13, 17, 28]. In contrast, Kurtz et al. [17] described an increase in crystallinity when annealing PE below its melting point after irradiation, but in our study, the 3-D comparisons of XLPE and conventional PE only involved remelted XLPE inserts. Most of the deformation occurs early after implantation on ultrahigh-molecular-weight PE bearing surfaces [11, 18, 19], consistent with our finding of linear correlations between LOI and volume change in the XLPE and conventional PE inserts (Fig. 5). The resulting larger contact area in XLPE TKA inserts attributable to the reduced creep resistance may correlate to reduced stresses on the surface of the XLPE (compared with what would occur on a conventional PE surface). However, whether this increased deformation leads to a meaningful clinical advantage is unclear.

Approaches other than laser scanning exist to assess PE wear. For example, knee simulator studies use gravimetric methods to measure the amount of PE worn from the articulating surfaces $[12,22,33,34]$. Of course this approach cannot be applied to retrieved implants because the original weights of the implants are unknown and the implants may show added weight through embedded thirdbody particles. Clinically, wear can be determined from serial radiographs by measuring the penetrating depth of the opposing metallic implant into the polyethylene surface. Although not as widely adapted for knee arthroplasty as for hip arthroplasty, the technique has been used to measure wear rates as a function of polyethylene sterilization techniques [7] and to predict TKA failure [9]. Of course the technique relies on the availability of high-quality, serial radiographs, which were unavailable for our study. 


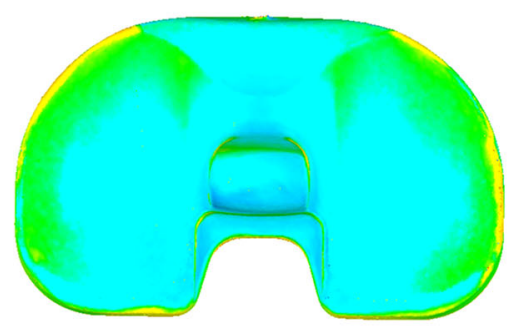

A

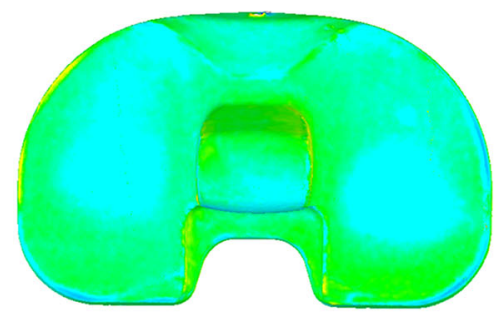

D

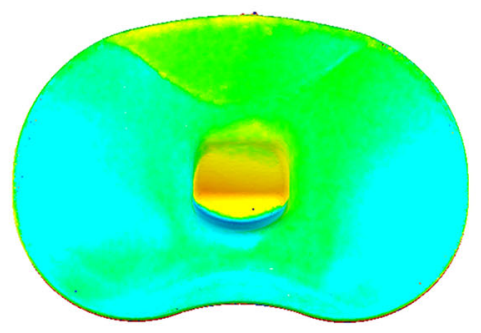

B

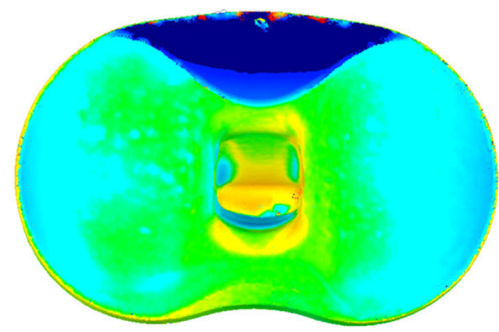

E

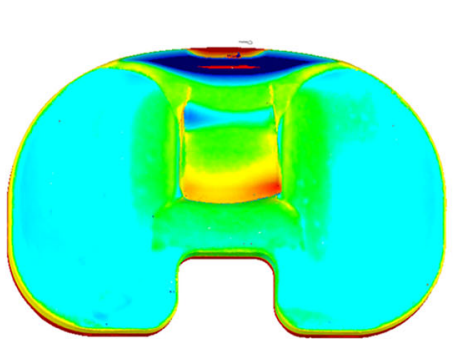

C

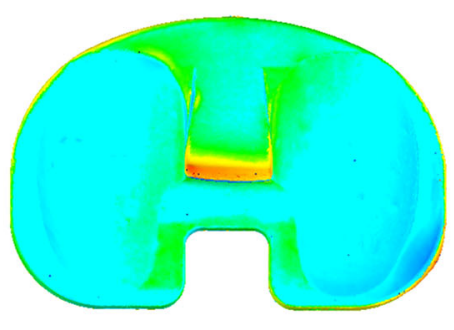

$\mathbf{F}$

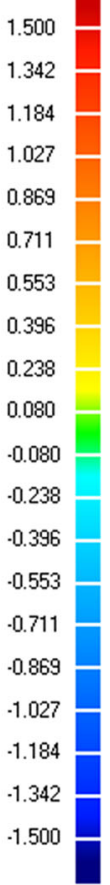

Fig. 6A-F Similar damage patterns were observed for the XLPE (AC) and conventional PE inserts (D-F). Negative deviations were mostly located on the center of the plateaus in retrieved Zimmer posterior-stabilized (PS) inserts (A, D), on the outer half of the plateaus in retrieved Smith \& Nephew PS inserts $(\mathbf{B}, \mathbf{E})$, and across the entire articular surface of the plateaus in retrieved DePuy PS inserts $(\mathbf{C}, \mathbf{F})$. Units are in millimeters.

3. Bartel DL, Bicknell VL, Wright TM. The effect of conformity, thickness, and material on stresses in ultra-high molecular weight components for total joint replacement. J Bone Joint Surg Am. 1986;68:1041-1051.

4. Bartel DL, Rawlinson JJ, Burstein AH, Ranawat CS, Flynn WF Jr. Stresses in polyethylene components of contemporary total knee replacements. Clin Orthop Relat Res. 1995;317:76-82.

5. Bragdon CR, Doerner M, Martell J, Jarrett B, Palm H, Malchau H. The 2012 John Charnley Award: Clinical multicenter studies of the wear performance of highly crosslinked remelted polyethylene in THA. Clin Orthop Relat Res. 2013;471:393402.

6. Capello WN, D'Antonio JA, Ramakrishnan R, Naughton M. Continued improved wear with an annealed highly cross-linked polyethylene. Clin Orthop Relat Res. 2011;469:825-830.

7. Collier MB, Engh CA Jr, Hatten KM, Ginn SD, Sheils TM, Engh GA. Radiographic assessment of the thickness lost from polyethylene tibial inserts that had been sterilized differently. $J$ Bone Joint Surg Am. 2008;90:1543-1552.

8. Cottrell JM, Townsend E, Lipman J, Sculco TP, Wright TM. Bearing surface design changes affect contact patterns in total knee arthroplasty. Clin Orthop Relat Res. 2007;464:127-131.

9. Engh CA Jr, Collier MB, Hopper RH Jr, Hatten KM, Engh GA. Radiographically measured total knee wear is constant and predicts failure. J Arthroplasty. 2013;28:1338-1344.

10. Engh CA Jr, Hopper RH Jr, Huynh C, Ho H, Sritulanondha S, Engh CA Sr. A prospective, randomized study of cross-linked and non-cross-linked polyethylene for total hip arthroplasty at 10year follow-up. J Arthroplasty. 2012;27:2-7.e1.

11. Estok DM 2nd, Bragdon CR, Plank GR, Huang A, Muratoglu OK, Harris WH. The measurement of creep in ultrahigh molecular weight polyethylene: a comparison of conventional versus 
highly cross-linked polyethylene. J Arthroplasty. 2005;20:239243.

12. Fisher J, McEwen HM, Tipper JL, Galvin AL, Ingram J, Kamali A, Stone MH, Ingham E. Wear, debris, and biologic activity of cross-linked polyethylene in the knee: benefits and potential concerns. Clin Orthop Relat Res. 2004;428:114-119.

13. Gencur SJ, Rimnac CM, Kurtz SM. Failure micromechanisms during uniaxial tensile fracture of conventional and highly crosslinked ultra-high molecular weight polyethylenes used in total joint replacements. Biomaterials. 2003;24:3947-3954.

14. Gencur SJ, Rimnac CM, Kurtz SM. Fatigue crack propagation resistance of virgin and highly crosslinked, thermally treated ultra-high molecular weight polyethylene. Biomaterials. 2006; 27:1550-1557.

15. Hood RW, Wright TM, Burstein AH. Retrieval analysis of total knee prostheses: a method and its application to 48 total condylar prostheses. J Biomed Mater Res. 1983;17:829-842.

16. Inacio MC, Cafri G, Paxton EW, Kurtz SM, Namba RS. Alternative bearings in total knee arthroplasty: risk of early revision compared to traditional bearings: an analysis of 62,177 primary cases. Acta Orthop. 2013;84:145-152.

17. Kurtz SM, Villarraga ML, Herr MP, Bergstrom JS, Rimnac CM, Edidin AA. Thermomechanical behavior of virgin and highly crosslinked ultra-high molecular weight polyethylene used in total joint replacements. Biomaterials. 2002;23:3681-3697.

18. Lee KY, Pienkowski D. Compressive creep characteristics of extruded ultrahigh-molecular-weight polyethylene. J Biomed Mater Res. 1998;39:261-265.

19. Lewis G, Carroll M. Effect of crosslinking UHMWPE on its tensile and compressive creep performance. Biomed Mater Eng. 2001;11:167-183.

20. MacDonald DW, Higgs G, Parvizi J, Klein G, Hartzband M, Levine H, Kraay M, Rimnac CM, Kurtz SM. Oxidative properties and surface damage mechanisms of remelted highly crosslinked polyethylenes in total knee arthroplasty. Int Orthop. 2013; 37:611-615.

21. McCalden RW, MacDonald SJ, Rorabeck CH, Bourne RB, Chess DG, Charron KD. Wear rate of highly cross-linked polyethylene in total hip arthroplasty. A randomized controlled trial. $J$ Bone Joint Surg Am. 2009;91:773-782.

22. Muratoglu OK, Rubash HE, Bragdon CR, Burroughs BR, Huang A, Harris WH. Simulated normal gait wear testing of a highly cross-linked polyethylene tibial insert. J Arthroplasty. 2007; 22:435-444.
23. Muratoglu OK, Ruberti J, Melotti S, Spiegelberg SH, Greenbaum ES, Harris WH. Optical analysis of surface changes on early retrievals of highly cross-linked and conventional polyethylene tibial inserts. J Arthroplasty. 2003;18:42-47.

24. Mutimer J, Devane PA, Adams K, Horne JG. Highly crosslinked polyethylene reduces wear in total hip arthroplasty at 5 years. Clin Orthop Relat Res. 2010;468:3228-3233.

25. Oral E, Malhi AS, Muratoglu OK. Mechanisms of decrease in fatigue crack propagation resistance in irradiated and melted UHMWPE. Biomaterials. 2006;27:917-925.

26. Ranawat AS, Tsailis P, Meftah M, Koob TW, Rodriguez JA, Ranawat CS. Minimum 5-year wear analysis of first-generation highly cross-linked polyethylene in patients 65 years and younger. J Arthroplasty. 2012;27:354-357.

27. Reinitz SD, Currier BH, Levine RA, Van Citters DW. Crosslink density, oxidation and chain scission in retrieved, highly cross-linked UHMWPE tibial bearings. Biomaterials. 2014;35:4436-4440.

28. Ries MD, Pruitt L. Effect of cross-linking on the microstructure and mechanical properties of ultra-high molecular weight polyethylene. Clin Orthop Relat Res. 2005;440:149-156.

29. Rimnac C, Pruitt L. How do material properties influence wear and fracture mechanisms? J Am Acad Orthop Surg. 2008; 16(Suppl 1):S94-100.

30. Sakellariou VI, Sculco P, Poultsides L, Wright T, Sculco TP. Highly cross-linked polyethylene may not have an advantage in total knee arthroplasty. HSS J. 2013;9:264-269.

31. Stoner KE, Nassif NA, Wright TM, Padgett DE. Laser scanning as a useful tool in implant retrieval analysis: a demonstration using rotating platform and fixed bearing tibial inserts. $J$ Arthroplasty. 2013;28:152-156.

32. Thomas GE, Simpson DJ, Mehmood S, Taylor A, McLardy-Smith P, Gill HS, Murray DW, Glyn-Jones S. The seven-year wear of highly cross-linked polyethylene in total hip arthroplasty: a doubleblind, randomized controlled trial using radiostereometric analysis. J Bone Joint Surg Am. 2011;93:716-722.

33. Utzschneider S, Harrasser N, Sadoghi P, Weber P, Schroder C, Pietschmann MF, Jansson V. Crosslinked polyethylene in knee arthroplasty: a simulator study evaluating the positive influence on the tribocontact area in the fixed-bearing knee. Arch Orthop Trauma Surg. 2010;130:1419-1424.

34. Utzschneider S, Harrasser N, Schroeder C, Mazoochian F, Jansson V. Wear of contemporary total knee replacements-a knee simulator study of six current designs. Clin Biomech (Bristol, Avon). 2009;24:583-588. 